\title{
VAMP-2, SNAP-25A/B and syntaxin-1 in glutamatergic and GABAergic synapses of the rat cerebellar cortex
}

\author{
Vincenzo Benagiano ${ }^{1 *}$, Loredana Lorusso ${ }^{1}$, Paolo Flace ${ }^{1}$, Francesco Girolamo ${ }^{1}$, Anna Rizzi ${ }^{1}$, Lorenzo Bosco ${ }^{2}$, \\ Raffaele Cagiano ${ }^{3}$, Beatrice Nico ${ }^{1}$, Domenico Ribatti ${ }^{1}$ and Glauco Ambrosi ${ }^{1}$
}

\begin{abstract}
Background: The aim of this study was to assess the distribution of key SNARE proteins in glutamatergic and GABAergic synapses of the adult rat cerebellar cortex using light microscopy immunohistochemical techniques. Analysis was made of co-localizations of vGluT-1 and vGluT-2, vesicular transporters of glutamate and markers of glutamatergic synapses, or GAD, the GABA synthetic enzyme and marker of GABAergic synapses, with VAMP-2, SNAP-25A/B and syntaxin-1.
\end{abstract}

Results: The examined SNARE proteins were found to be diffusely expressed in glutamatergic synapses, whereas they were rarely observed in GABAergic synapses. However, among glutamatergic synapses, subpopulations which did not contain VAMP-2, SNAP-25A/B and syntaxin-1 were detected. They included virtually all the synapses established by terminals of climbing fibres (immunoreactive for vGluT-2) and some synapses established by terminals of parallel and mossy fibres (immunoreactive for vGluT-1, and for vGluT-1 and 2, respectively). The only GABA synapses expressing the SNARE proteins studied were the synapses established by axon terminals of basket neurons.

Conclusion: The present study supplies a detailed morphological description of VAMP-2, SNAP-25A/B and syntaxin1 in the different types of glutamatergic and GABAergic synapses of the rat cerebellar cortex. The examined SNARE proteins characterize most of glutamatergic synapses and only one type of GABAergic synapses. In the subpopulations of glutamatergic and GABAergic synapses lacking the SNARE protein isoforms examined, alternative mechanisms for regulating trafficking of synaptic vesicles may be hypothesized, possibly mediated by different isoforms or homologous proteins.

\section{Background}

According to the hypothesis of the soluble N-ethylmaleimide sensitive factor attachment receptor (SNARE), common molecular mechanisms exist in chemical synapses, that regulate the processes of docking, priming and fusion between the synaptic vesicle membrane and specific regions of the plasma membrane (target membrane) and the subsequent release (exocytosis) of neurotransmitters. In the original formulation of the SNARE hypothesis, three proteins were considered, one localized on the vesicle membrane ( $\mathrm{v}$-SNARE), synaptobrevin or

\footnotetext{
* Correspondence: benagiano@anatomia.uniba.it

'Dipartimento Scienze Mediche di Base - Sezione di Anatomia e Istologia, Università Bari - 70124 Bari - Italy

Full list of author information is available at the end of the article
}

vesicular associated membrane protein (VAMP), and two localized on the target membrane (t-SNAREs), syntaxin and synaptosomal associated protein of $25 \mathrm{kDa}$ (SNAP-25) [1,2]. When a synaptic site is depolarized, calcium ions penetrate the site and trigger VAMP, syntaxin and SNAP-25 to bind together in a lock and key fashion to form a tripartite structure, the SNARE complex (or core). This serves as a receptor for the cytoplasmic ATP-ase protein, N-ethylmaleimide sensitive factor (NSF), and its soluble attachment proteins. Finally, ATP hydrolysis by NSF leads to exocytosis and induces a conformational change in the complex that results in its disassembly [3-9]. Several biochemical and immunohistochemical studies suggest the existence of SNARE mechanisms virtually in all regions of the CNS [10-15].

\section{Biomed Central}


Multiple isoforms and homologous proteins, originated by differential splicing of single genes or codified by different genes of the same family, respectively, have been described for VAMP [16], SNAP-25 [17-21] and syntaxin [18,22-25]. The different isoforms and homologous proteins display distinct patterns of distribution within CNS synapses, being present in one type of synapse and absent in another, and it has been hypothesized that when a SNARE protein is lacking, a different isoform or homologous protein will perform its function [26-29]. Therefore, SNARE mechanisms should be present in all chemical synapses, but the expression of SNARE proteins may vary. In particular, specific sets of SNARE proteins seem to be required to regulate trafficking of vesicles containing different types of neurotransmitters, possibly even one set per neurotransmitter [30,31]. According to this hypothesis, scholars have reported that SNARE proteins are strongly expressed in excitatory (glutamatergic) synapses and may be lacking in inhibitory (GABAergic) synapses [12,15,32-34].

The cerebellar cortex features a high concentration of synapses, mostly of two main types, namely glutamatergic synapses and GABAergic synapses, each type displaying distinct patterns of distribution within the cortical layers. In the molecular layer, the glutamatergic synapses are densely distributed in the neuropil, mainly consisting of terminals of parallel fibres and, to a lesser extent, of climbing fibres on the dendritic trees of Purkinje neurons [35-40]. In the same layer, GABAergic synapses are distributed throughout the neuropil and consist mainly of synapses of stellate neuron axon terminals on dendrite trees of Purkinje neurons [41-43]. In the Purkinje neuron layer, only GABAergic synapses are present, concentrated at the deep pole of the body of Purkinje neurons and formed by terminals of basket neuron axons [42-44]. In the granular layer, glutamatergic synapses and GABAergic synapses are both located in the neuropil regions scattered among granules (islands of Held), consisting of multiple synapses (glomeruli) established by glutamatergic terminals of mossy fibres and of axons of unipolar brush neurons $[35-38,45]$ and GABAergic axon terminals of Golgi, candelabrum and Lugaro neurons $[44,46,47]$, on granule dendrites.

Studies have reported widespread SNARE mechanisms at cerebellar cortex synapses $[11,13,34,48-54]$, but a detailed morphological description of the distribution of $\mathrm{v}$-SNARE and t-SNARE proteins in different types of glutamatergic and GABAergic synapses of the cerebellar cortex has not yet been supplied. Owing to the different patterns of distribution of glutamatergic and GABAergic synapses in the cerebellar cortex, it seems to be an ideal region where assess the distribution of $v$-SNARE and $t$ SNARE proteins within glutamatergic synapses and
GABAergic synapses. In the present study, using double labelling light microscopy immunohistochemistry techniques, we examined the cortical distribution of the colocalization of VAMP or SNAP-25 or syntaxin with markers of glutamatergic or GABAergic synapses. To reveal the SNARE proteins, we used antibodies directed against isoforms considered to be of crucial importance in the CNS synapses, namely VAMP-2 [55,56], SNAP$25 \mathrm{~A}$ and $\mathrm{B}[11,34]$ and syntaxin-1 $[32,57]$. The glutamatergic terminals were visualized by two different antibodies directed against the glutamate vesicular transporters, vGlut T-1 and vGlutT-2. This was necessary because vGluT-1 is expressed by terminals of parallel and mossy fibres, but not those of climbing fibres, and vGluT-2 is expressed by terminals of climbing and mossy fibres, but not those parallel fibres [58-62]. The GABAergic terminals were labelled using antibodies against isoforms 65 and 67 of glutamic acid decarboxylase (GAD), the enzyme involved in GABA synthesis, which labels axon terminals of GABAergic neurons of the cerebellar cortex, providing distribution patterns comparable to those of the vesicular GABA transporter (v-GAT) [63].

\section{Methods}

\section{Animals and samples}

Experiments were conducted on Wistar adult rats (Harlan SRC, Milano, Italy), aged 3 months, weighting 350$450 \mathrm{~g}$, and housed in accordance with the Italian Ministry of Health guidelines (D.L. 116/92 and D.L. 111/94B), the Declaration of Helsinki and National Institute of Health guidelines for the care and use of laboratory animals.

The animals were anaesthetized with equithesin $(3 \mathrm{ml}$ $\mathrm{kg}^{-1}$, injected i.p.) and transcardially perfused with a fixative solution containing $10 \%$ formaldehyde, $1 \%$ picric acid and $1 \%$ glutaraldehyde.

Brains were removed from the skull, and cerebella were isolated, sectioned in two parts on the median sagittal plane and postfixed by immersion in the same fixative solution for $1.5 \mathrm{~h}$ at $4^{\circ} \mathrm{C}$. Each hemicerebellum was dehydrated in an ethanol series, embedded in paraffin and serially cut into $5 \mu \mathrm{m}$ sagittal sections.

From each series of sections of cerebellar cortex, consecutive sections were chosen at intervals of $200 \mu \mathrm{m}$, in order to analyze all lobules of anterior and posterior lobe of cerebellum, and subjected to the following distributional analyses using immunofluorescence techniques: i. vGluT-1 and vGluT-2; ii. vGluT-1 and VAMP-2; iii. vGluT-1 and SNAP-25A/B; iv. vGluT-1 and syntaxin-1; v. vGluT-2 and VAMP-2; vi. vGluT-2 and SNAP-25A/B; vii. vGluT-2 and syntaxin-1; viii. GAD-65/67 and VAMP-2; ix. GAD-65/67 and SNAP-25A/B; x. GAD-65/ 67 and syntaxin-1. 


\section{Immunohistochemistry Antibodies}

The following commercial antibodies were used: 1 . antivGluT-1 polyclonal antibody, raised in guinea pig against a synthetic linear peptide from rat vGluT-1 (Millipore, Billerica, MA, USA) [34,64]; 2. anti-vGluT-2 polyclonal antibody, raised in rabbit against a strep-tag fusion protein of rat vGluT-2 (Synaptic System, Goettingen, Germany) [34,65]; 3. (a) anti-GAD monoclonal antibody, raised in mouse against human GAD-65-GST fusion protein (Stressgen, Victoria, BC, Canada), and (b) anti-GAD polyclonal antibody, raised in rabbit against a synthetic peptide corresponding to the rat GAD-65 Cterminus residues 572-585 (Chemicon), which react to both GAD-65 and GAD-67 [63]; 4. (a) anti-VAMP monoclonal antibody, raised in mouse against crude synaptic immunoprecipitate (human) (Millipore), and (b) anti-VAMP polyclonal antibody, raised in rabbit against a synthetic peptide based on rat VAMP-2 (Stressgen), which react to VAMP-2 [48]; 5. anti-SNAP25 monoclonal antibody, raised in against crude synaptic immunoprecipitate (human), which reacts with both SNAP-25A and SNAP-25B (Chemicon) [15]; 6. anti-syntaxin monoclonal antibody, raised in mouse against clone HCP-1, which reacts to syntaxin-1 (ABCAM, Cambridge, UK) [57].

\section{Immunolabelling procedures}

The sections were deparaffinized, rehydrated and immunolabelled as follows. 1. Immersion in sodium citrate buffer (0.01M, pH 6.0) for $3 \times 5$ min in microwave oven at $700 \mathrm{~W}$, to unmask antigens. 2. Treatment for $60 \mathrm{~min}$ at room temperature (RT) with a blocking solution containing $1 \%$ bovine serum albumin, 5\% fetal calf serum and $10 \%$ normal serum in sodium phosphate buffer

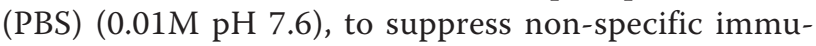
nolabelling. 3. Incubations overnight at $4^{\circ} \mathrm{C}$ with the primary antibodies at the following dilutions in blocking solution: (a) vGluT-1 (1:400) and vGluT-2 (1:400); (b) vGluT-1 (1:400) and VAMP-2 (rabbit, 1:300); (c) vGluT1 (1:400) and SNAP-25A/B (1:800); (d) vGluT-1 (1:400) and syntaxin-1 (1:50); (e) vGluT-2 (1:400) and VAMP-2 (mouse, 1:100); (f) vGluT-2 (1:400) and SNAP-25A/B (1:800); (g) vGluT-2 (1:400) and syntaxin-1 (1:50); (h) GAD-65/67 (mouse, 1:100) and VAMP-2 (rabbit, 1:300); (i) GAD-65/67 (rabbit, 1:100) and SNAP-25A/B (1:800); (j) GAD-65/67 (rabbit, 1:100) and syntaxin-1 (1:50). 4. Incubations for $60 \mathrm{~min}$ at RT with the secondary antibodies diluted in PBS: (i) alexa fluor 568 anti-rabbit antibody (goat, 1:100) (Molecular Probes); (ii) alexa fluor 568 anti-mouse antibody (goat, 1:100) (Molecular Probes); (iii) alexa fluor 568 anti-guinea pig antibody (goat, 1:100) (Molecular Probes); (iv) alexa fluor 488 fragment-conjugated anti-rabbit antibody (goat, 1:100) (Molecular Probes, Invitrogen Corporation, Carlsbad,
CA, USA); (v) alexa fluor 488 anti-mouse antibody (goat, 1:100) (Molecular Probes); (vi) alexa fluor 488 anti-guinea pig antibody (goat, 1:100) (Molecular Probes); (vii) biotinylated anti-rabbit antibody (donkey, 1:100) (Santa-Cruz Biotechnology Inc., Santa Cruz, CA, USA). 5. Incubation of the sections treated with the biotinylated secondary anti-rabbit antibody for $60 \mathrm{~min}$ at $\mathrm{RT}$ in the fluorescein-avidin D solution (Vector Laboratories, Burlingame, CA, USA), diluted $5 \mu \mathrm{g} / \mathrm{ml}$ in PBS. 6. Mounting of sections by Vectashield (Vector).

All observations were carried out under the light microscope Axioskop (Zeiss, Germany) equipped with a high-resolution color video camera (Spot Insight, Diagnostic Instruments Inc., MI, USA).

\section{Results}

The patterns of distribution of immunoreactivities for all the markers examined in this study appeared to be comparable in the different lobes and lobules of cerebellum.

vGluT-1 and vGluT-2, VAMP-2, SNAP-25A/B and syntaxin-1 Immunohistochemical techniques for vGluT-1 and vGluT-2 produced a punctate staining, attributable to the three main systems of glutamatergic terminals in the cerebellar cortex (Figure 1, Figure 2, Figure 3, Figure 4). Precisely, immunohistochemistry for vGluT-1 revealed: in the molecular/Purkinje layer (ML), the terminals of parallel fibres, which were densely and homogeneously distributed in the abundant neuropil interposed among bodies and processes of stellate, basket, Purkinje neurons and blood vessels (Figure 1a); in the granular layer (GL), the terminals of mossy fibres, which were localized in discrete regions of the neuropil comprised among granules (Figure 1b). Immunohistochemistry for vGluT2 revealed: in the ML, the terminals of climbing fibres, localized in the inner zone of the layer, where they formed discrete rows surrounding body and dendrite trunks of Purkinje neurons (Figure 1a); in the GL, the terminals of mossy fibres, distributed in the neuropil with a pattern similar to that of vGluT-1 immunoreactivity (Figure 1b). Double labelling for vGluT-1 and vGluT-2 revealed co-localization of vGluT-1 and vGluT2 only in the granular layer, in a subpopulation of terminals of mossy fibres (Figure 1b).

Immunoreactions for VAMP-2, SNAP-25A/B and syntaxin-1 produced a diffuse staining in the cortex. The qualitative patterns of distribution of the three examined SNARE were similar. The immunoreactivities were observed in fine, highly concentrated dots, which almost completely filled the neuropil of the whole cortex ('background of positivity'), whereas the neuronal bodies and processes were constantly negative to the immunoreactions (Figure 2, Figure 3, Figure 4, Figure 5, Figure 6). 


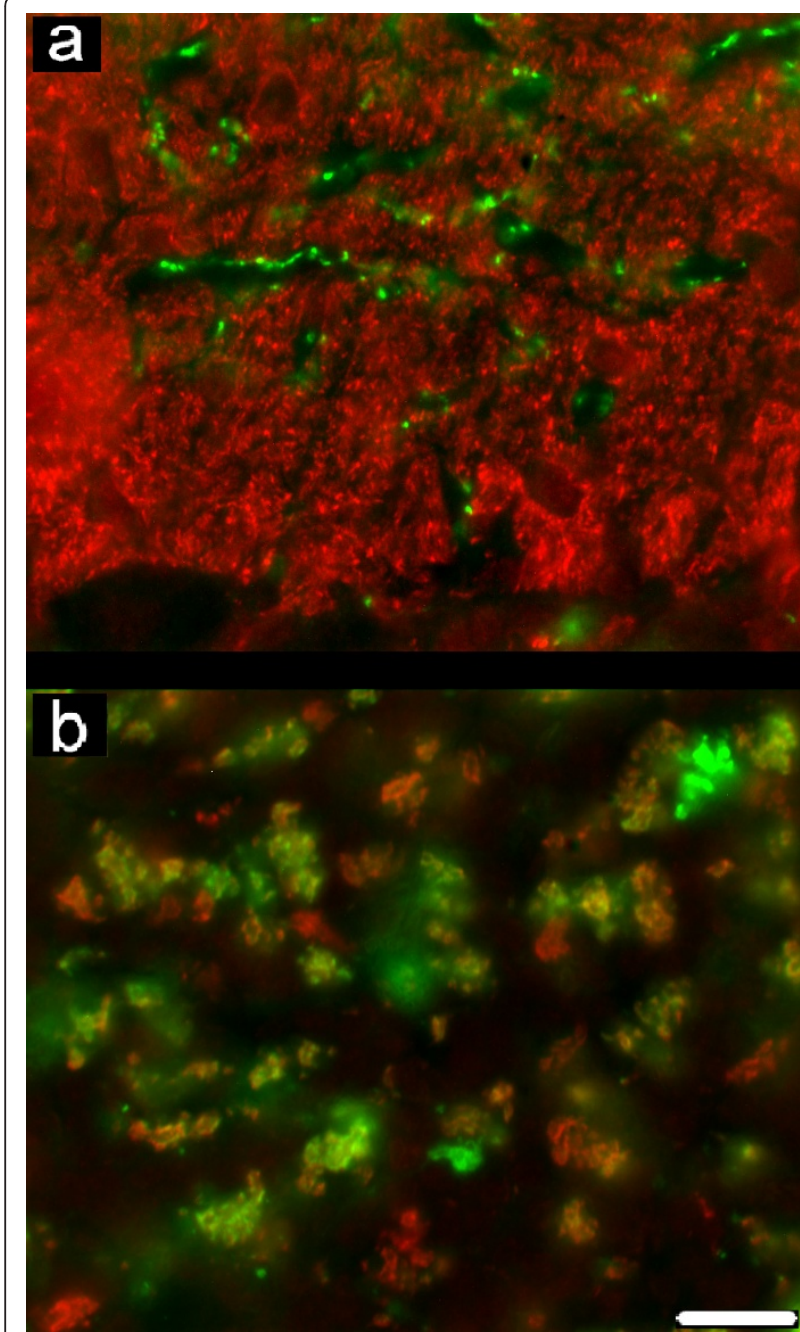

Figure 1 Double labelling for vGluT-1 and vGluT-2. (a) In the molecular layer, puncta immulabelled for vGluT-1 (green) are densely distributed in the neuropil; puncta immulabelled for vGluT-2 (red) are localized in the inner zone of the layer, in anatomical relation to body and dendrite trunks of Purkinje neurons. (b) In the granular layer, puncta double immunolabelled for vGluT-1 and vGluT-2 (yellow), and puncta immunoreactive for vGluT-1 or VGluT-2 are present. Scale bar: $10 \mu \mathrm{m}$.

Double labelling for vGluT-1 and VAMP-2 (Figure 2c, Figure 2f) or SNAP-25A/B (Figure 2i, Figure 2l) or syntaxin-1 (Figure 2o, Figure 2r; Figure 3) revealed numerous punctate elements in the ML and GL, which displayed a co-localization of vGluT-1 with VAMP-2 or with SNAP-25A/B or with syntaxin-1. The double labelled puncta were seen as zones of focal immunoreactivity standing out clearly against the background of positivity provided by the immunoreactivity for the three SNARE proteins. However, in all the layers, a number of vGluT-1-immunoreactive puncta were immunonegative for VAMP-2, SNAP-25A/B and syntaxin-1. Double labelling for vGluT-2 and VAMP-2 (Figure 4c) or SNAP-25A/B (Figure 4f) or syntaxin-1 (Figure 4i) revealed the absence of any co-localization in the ML. Instead, in the GL, a number puncta were observed to co-localize vGluT-2 with the examined SNARE proteins (Figure 4c, Figure 4f, Figure 4i).

\section{GAD-65/67, VAMP-2, SNAP-25A/B and syntaxin-1}

Immunohistochemistry for GAD-65/67 revealed punctate elements, putative GABAergic axon terminals, as well as neuronal bodies and processes, distributed throughout the cortex with layer specific patterns of distribution (Figure 5a, Figure 5d, Figure 5g).

In the double labelling for GAD-65/67 and VAMP-2 (Figure 5c) or SNAP-25A/B (Figure 5f) or syntaxin-1 (Figure 5i; Figure 6), the GAD-65/67-immunoreactive elements appeared as areas of focal positivity against the background of positivity displayed by VAMP-2 or SNAP-25A/B or syntaxin-1 immunoreactivities. Some puncta at the deep pole of the Purkinje neuron body, where are concentrated the axon terminals of basket neurons (pinceau), displayed a co-localization of GAD65/67 and the SNARE proteins studied (Figure 5c,Figure 5f, Figure 5i; Figure 6b). No co-localization was observed in the GL (Figure 5c, Figure 5f, Figure 5i; Figure 6c).

\section{Discussion}

The demonstration in the rat cerebellar cortex of diffuse immunoreactivity for VAMP (isoform 2), SNAP-25 (isoforms $\mathrm{A}$ and $\mathrm{B}$ ) and syntaxin (isoform 1), morphologically confirms a massive involvement of SNARE mechanisms in the regulation of trafficking of synaptic vesicles and release of neurotransmitters at cortex synapses $[11,13,48-51,57]$. The SNARE proteins examined were found densely distributed in the neuropil of all layers of the cerebellar cortex. However, the characteristic background of positivity displayed by the proteins indicates that they are not localized exclusively in synaptic terminals, but ubiquitously in neuronal processes until their more distal ramifications. This observation agrees with the assumption that SNARE proteins may be involved also in non-synaptic functions, such as the regulation of morphogenic processes (axon elongation, synapse formation, receptor recycling) [57,66-68]. Moreover, the presence of these proteins in non-synaptic regions could indicate that mechanisms of vesicle exocytosis may occur not only at synapses, but also outside of them, along the whole neuronal membrane. This observation could constitute the morphological basis of an extrasynaptic release of neurotransmitter, which act diffusely within a volume of nervous tissue (volume transmission) rather than at the level of a certain number of spatially separate synapses. 


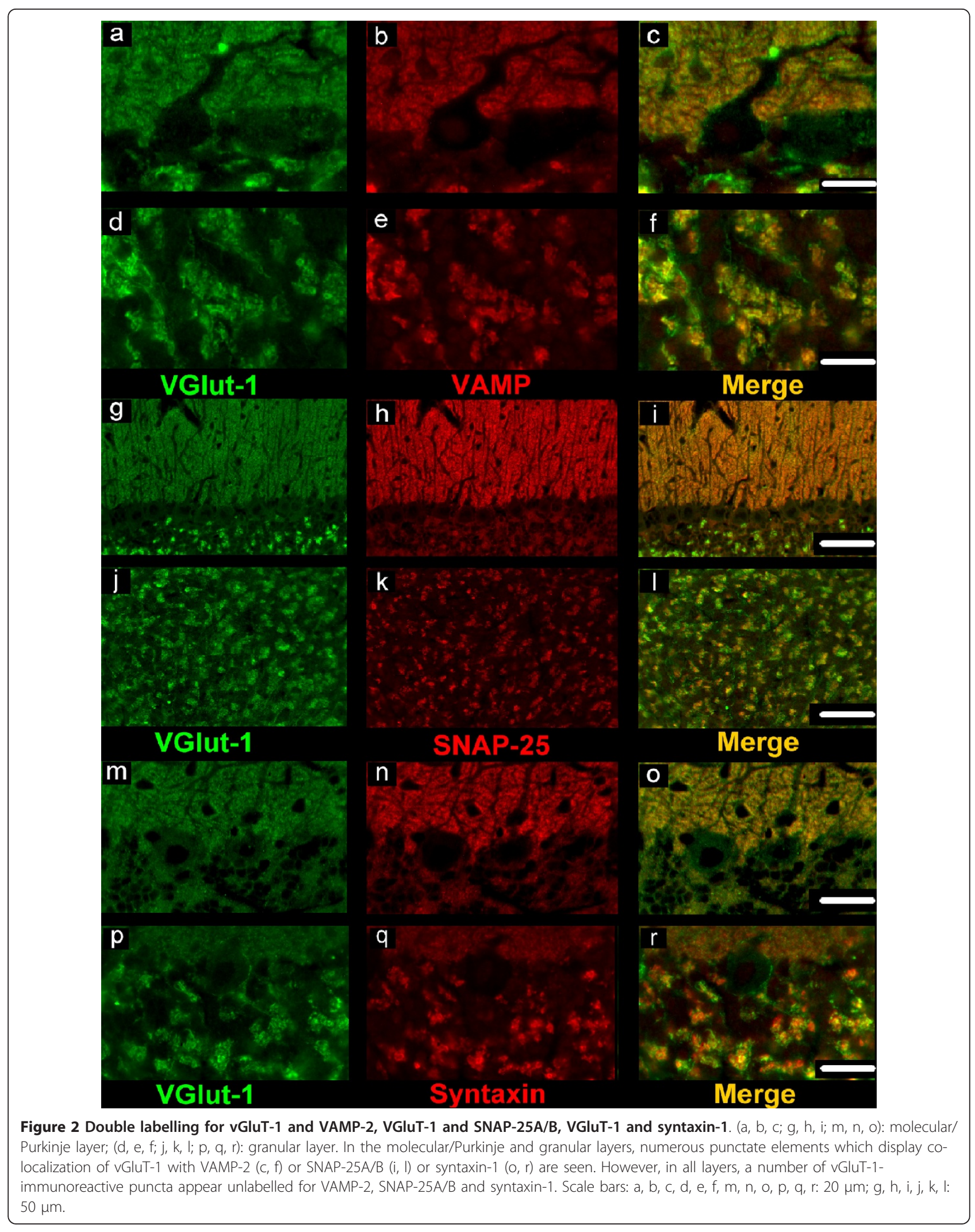



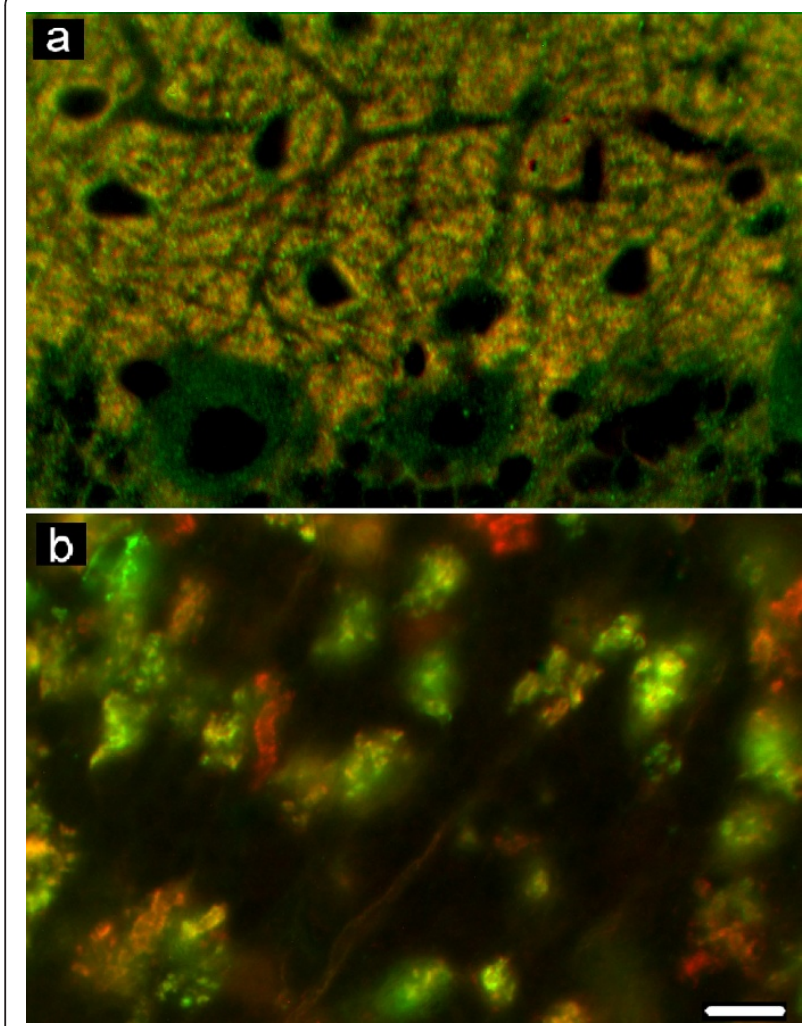

Figure 3 Double labelling for vGluT-1 and syntaxin-1. In (a) the molecular layer and (b) granular layer, double labelled punctate elements, putative axon terminals belonging to parallel fibres and mossy fibres, respectively, are seen. In both the layers,

subpopulation of terminals positive only for vGluT-1 are present. Scale bar: $10 \mu \mathrm{m}$

The novelty of this study derives mainly from results obtained by double labelling experiments for the SNARE proteins and markers of glutamatergic (vGluT-1 and vGluT-2) and GABAergic (GAD-65/67) synapses. These experiments allowed us to recognize the glutamatergic or GABAergic axon terminals which contain the examined SNARE proteins or not. In fact, the immunoreactivities for VAMP-2, SNAP-25A/B and syntaxin-1, widely distributed in the cortex, do not provide precise information about the synapses in which they are expressed. On the contrary, the focal immunoreactivity for vGluT-1 and vGluT-2, and for GAD-65/67, owing to their well-defined distributional patterns in the cerebellar cortex, can be useful to recognize the glutamatergic synapses and GABAergic synapses, respectively. The results evidence, among the glutamatergic and GABAergic synapses of the cerebellar cortex, subpopulations which express the SNARE proteins and subpopulations which do not express them.

\section{Glutamatergic synapses}

The glutamatergic synapses of the cerebellar cortex were recognized on the basis of the topographic distribution in the cerebellar cortex layers and expression of vesicular glutamate transporters, vGluT-1 and/or vGluT-2 [39,40,58-62]. In the present study, we observed that the glutamatergic synapses between the parallel fibre terminals and the dendritic tree of Purkinje neurons frequently display co-localization of vGluT-1 with the three SNARE proteins examined. On the contrary, the glutamatergic synapses between the climbing fibre terminals and the body and proximal part of dendrite tree of Purkinje neurons express vGluT-2, but are negative for the SNARE proteins. In the granular layer, most of the glutamatergic terminals of the mossy fibre and of unipolar brush neuron axon appear immunoreactive for vGluT-1 or vGluT-2 and for the three SNARE proteins examined. However, some terminals of parallel fibres (vGluT-1 immunoreactive) and mossy fibres (vGluT-1 and/or vGluT-2 immunoreactive) do not express VAMP-2, SNAP-25A/B and syntaxin-1.

These data agree with the assumption that the SNARE mechanisms largely characterize the excitatory synapses and that glutamate-containing vesicles release their content via SNARE-dependent mechanisms [12,15,33,34]. However, this study demonstrates subpopulations of glutamatergic synapses in the cerebellar cortex that do not contain the examined SNARE proteins. They include virtually all synapses from terminals of climbing fibres, but also some of synapses from parallel and mossy fibres. The terminals positive for vGluT-1 and vGluT-2, but negative for VAMP-2, SNAP-25A/B and syntaxin-1 could be glutamatergic terminals caught in a functional phase which do not express detectable levels of SNARE proteins. Alternatively, they could be cross-sectioned glial processes. In fact, studies have reported that processes of astrocytes may express vesicular glutamate transporters $[69,70]$, but never express SNARE proteins (at least the isoforms examined in this study) [71].

\section{GABAergic synapses}

The GABAergic synapses in the molecular layer, mainly represented by the synapses between the axon terminals of stellate neurons and dendritic tree of Purkinje neurons, express GAD-65/67, but are constantly immunonegative for the three SNARE proteins examined. The GABAergic synapses in the granular layer, mainly represented by the synapses between the axon terminals of Golgi, candelabrum and Lugaro neurons and dendrites of granules, express GAD-65/67, but do not express immunoreactivity for the examined SNARE proteins. The only elements which co-localize GAD-65/67 with VAMP-2 or SNAP-25A/B or syntaxin-1 are represented by some axon terminals of basket neurons, making synapse on the deep pole of the Purkinje neurons body.

These results indicate that, in most GABAergic synapses of the cerebellar cortex, VAMP-2, SNAP- 

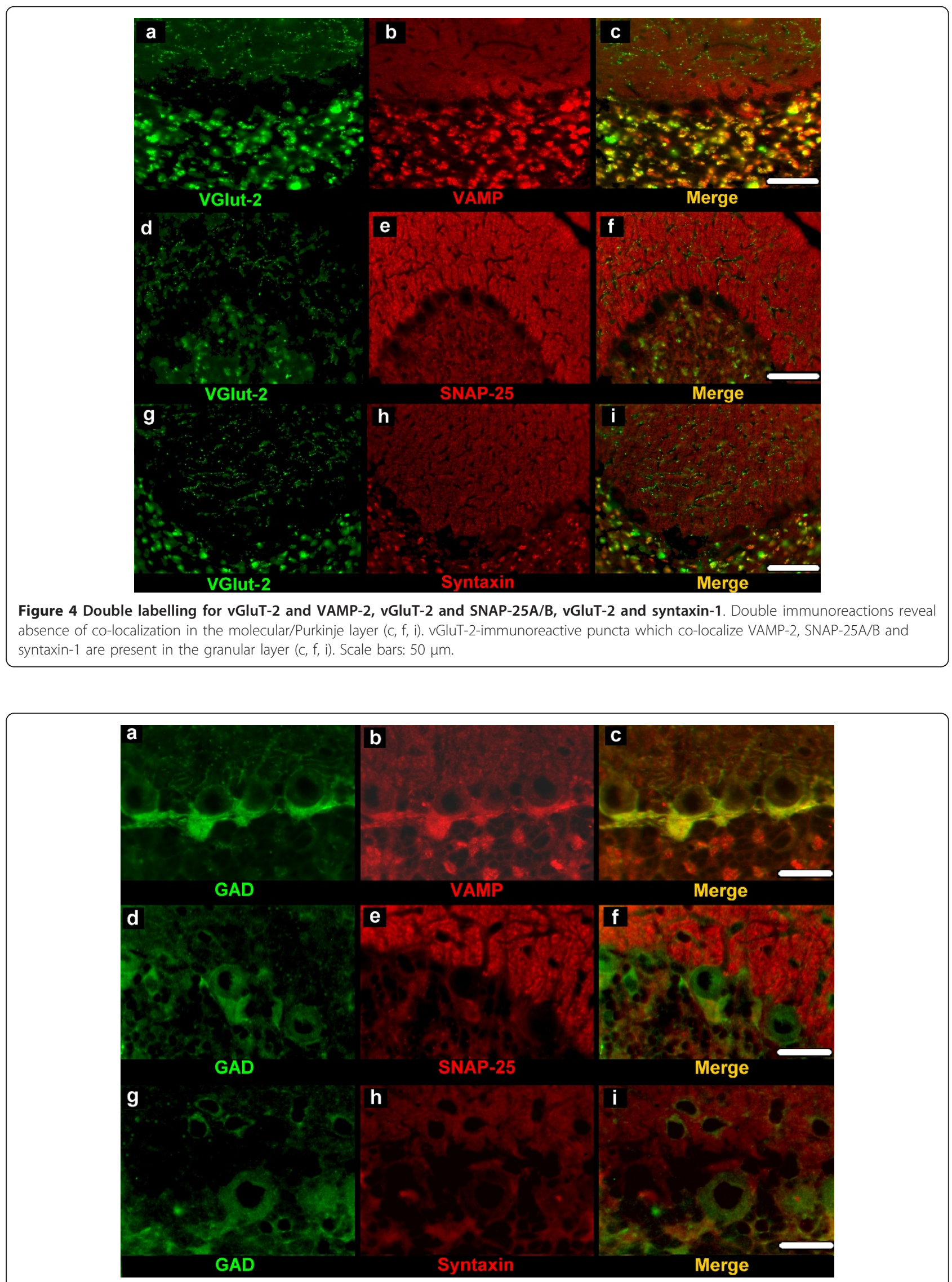

Figure 5 Double labelling for GAD-65/67 and VAMP-2, GAD-65/67 and SNAP-25A/B, GAD-65/67 and syntaxin-1. Co-localization is detectable only in puncta localized on the deep pole of the Purkinje neuron body (c, f, i). Scale bars: $20 \mu \mathrm{m}$. 
25A/B and syntaxin-1 do not participate in SNARE complexes responsible for trafficking of GABA-containing vesicles. This observation largely agrees with the assumption that SNAP-25 independent exocytotic pathways exist to support neurotransmission in GABAergic synapses [12,33], even if this assumption is not accepted by some researchers [14]. Moreover, it should again pointed out that some GABAergic axon terminals of basket neurons are exceptions to this assumption.

\section{Conclusions}

VAMP-2, SNAP-25A/B and syntaxin-1 are differently patterns distributed among the glutamatergic and GABAergic synapses of the adult rat cerebellar cortex. They largely characterize glutamatergic synapses, but lack in subpopulations of them, and are absent in most of GABAergic synapses, with the exception of some synapses between basket and Purkinje neurons.

In the subpopulations of glutamatergic and GABAergic synapses that do not express VAMP-2, SNAP-25A/B and syntaxin-1, alternative mechanisms regulating the exocytosis of glutamate or GABA neurotransmitters may be hypothesized, possibly based on the presence of homologous proteins. The SNARE mechanisms should be present in cerebellar cortex synapses, but in the synapse subpopulations that lack VAMP-2, SNAP-25A/ $B$ and syntaxin-1, they should be supported by other proteins, which are able to replace the missing ones, even though they were not labelled by the antibodies used in this study. This hypothesis is supported by the demonstration in the mammalian cerebellar cortex of a number of isoform and homologous proteins different from VAMP-2, SNAP-25A/B and syntaxin-1 (e.g., VAMP-1; SNAP-23, SNAP-47; syntaxin-3A, B, C, D) $[13,21,24,34]$.

Since the presence, or the absence, of co-localizations of markers of glutamate or GABA with the key SNARE proteins examined in this study must be unequivocally established, the present results need to be integrated by further qualitative and quantitative studies carried out using confocal and electron microscopy. The elucidation of the molecular features of the glutamatergic and GABAergic synapses in the cerebellar cortex, and in particular of the mechanisms underlying the interactions between vesicle membrane and presynaptic membrane in these synapses, will be important not only to obtain further insights on the chemical and morphofunctional organization of cerebellar cortex synapses, but also for possible impact in clinical medicine (e.g., for the identification of potential targets for drug therapies that may act selectively at cerebellar glutamatergic or GABAergic synapses).

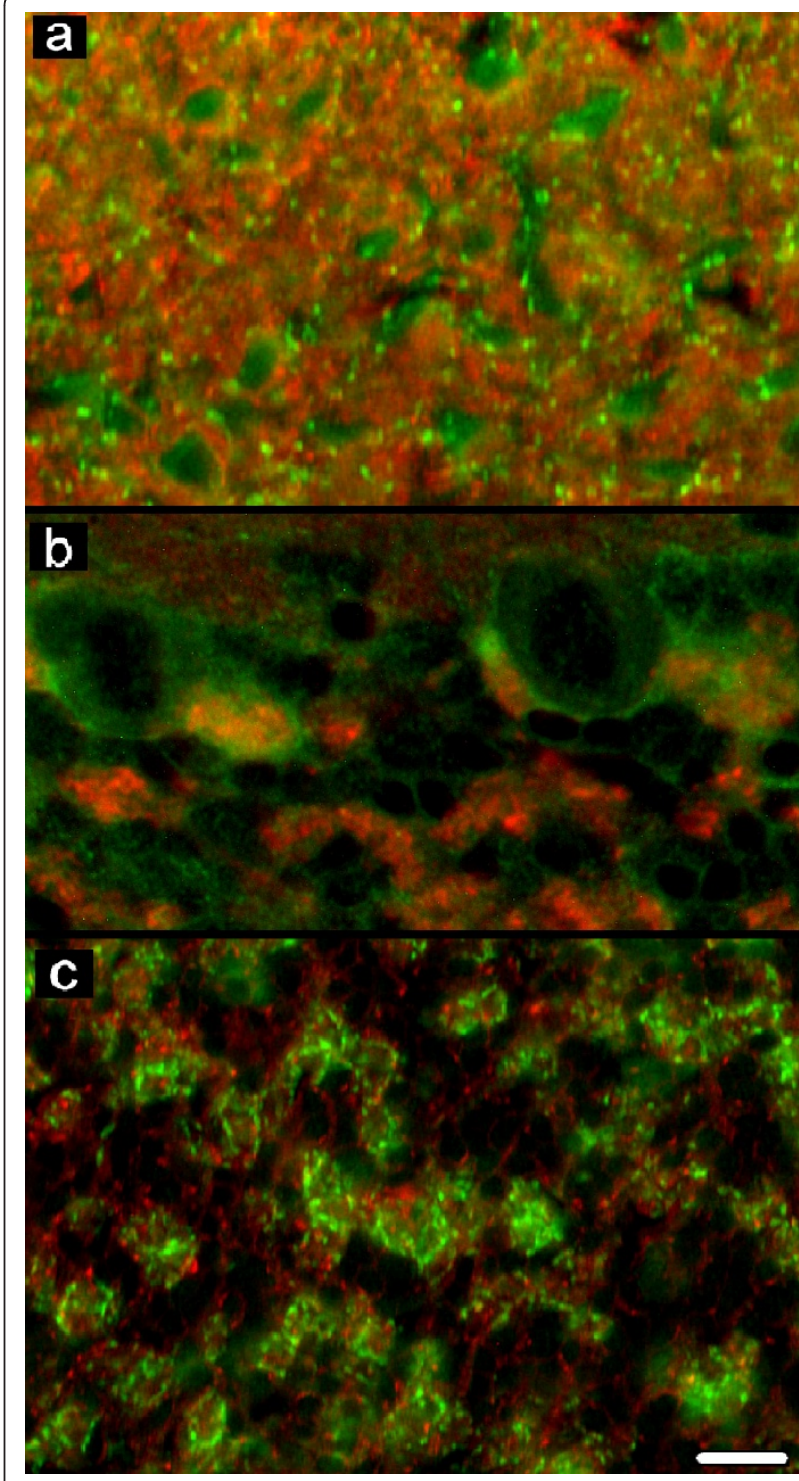

Figure 6 Double labelling for GAD-65/67 and syntaxin-1. In (a) the molecular and $(b, c)$ granular layer, puncta co-localizing GAD65/67 and syntaxin-1 are absent. Puncta which co-localize GAD-65/ 67 and syntaxin-1 are seen only on the deep pole of Purkinje neuron bodies (b). Scale bar: $10 \mu \mathrm{m}$.

\section{Acknowledgments and funding}

The authors are grateful to Ms. Mary Victoria Pragnell, BA, for linguistic assistance, and Mr. Francesco Fumai for technical assistance.

The study was supported by grants from the University of Bari (Italy) to G. Ambrosi.

\section{Author details}

'Dipartimento Scienze Mediche di Base - Sezione di Anatomia e Istologia, Università Bari - 70124 Bari - Italy. ${ }^{2}$ Dipartimento Bioetica, Università Bari 70124 Bari - Italy. ${ }^{3}$ Dipartimento Scienze Biomediche e Oncologia Umana Sezione Farmacologia, Università Bari - 70124 Bari - Italy.

\section{Authors' contributions}

VB and LL equally contributed to this study, designed the study, carried out experiments, collected data, draft the manuscript. PF carried out 
experiments, collected data. FG, AR, LB, RC analyzed and discussed the results. BN and DR analyzed and discussed the results, revised the manuscript. GA designed the study, co-ordinated the laboratory activities, analyzed and discussed the results, revised the manuscript.

All authors read and approved the final manuscript. The authors declare that they have no competing interests.

Received: 19 August 2011 Accepted: 17 November 2011 Published: 17 November 2011

\section{References}

1. Sollner T, Whiteheart SW, Brunner M, Erdjument-Bromage H, Geromanos S, Tempst $P$, Rothman JE: SNAP receptors implicated in vesicle targeting and fusion. Nature 1993, 362:318-324.

2. Rothman JE, Warren G: Implications of the SNARE hypothesis for intracellular membrane topology and dynamics. Curr Biol 1994, 4:220-233.

3. Otto H, Hanson Pl, Jahn R: Assembly and disassembly of a ternary complex of synaptobrevin, syntaxin, and SNAP-25 in the membrane of synaptic vesicles. Proc Natl Acad Sci USA 1997, 94:6197-6201.

4. Bock JB, Scheller RH: SNARE proteins mediate lipid bilayer fusion. Proc Natl Acad Sci USA 1999, 96:12227-12229.

5. Scales SJ, Bock JB, Scheller RH: The specifics of membrane fusion. Nature 2000, 407:144-146.

6. Chen YA, Scheller RH: SNARE-mediated membrane fusion. Nat Rev Mol Cell Biol 2001, 2:98-106

7. Jahn R, Lang T, Sudhof TC: Membrane fusion. Cell 2003, 112:519-533.

8. Sudhof TC: The synaptic vesicle cycle. Annu Rev Neurosci 2004, 27:509-547.

9. Verhage M, Sorensen JB: Vesicle docking in regulated exocytosis. Traffic 2009, 9:1414-1424.

10. Aguado F, Majo G, Ruiz-Montasell B, Lorens J, Marsal J, Blasi J: Syntaxin 1A and $1 \mathrm{~B}$ display distinct distribution patterns in the rat peripheral nervous system. Neuroscience 1998, 88:437-446.

11. Chen D, Minger SL, Honer WG, Witheheart SW: Organization of the secretory machinery in the rodent brain. distribution of the t-SNAREs, SNAP-25 and SNAP-23. Brain Res 1999, 831:11-24.

12. Verderio $C$, Pozzi D, Pravettoni E, Inverardi F, Schenk U, Coco S, ProuxGillardeaux V, Galli T, Rossetto O, Frassoni C, Matteoli M: SNAP-25 modulation of calcium dynamics underlies differences in GABAergic and glutamatergic responsiveness to depolarization. Neuron 2004, 41:599-610.

13. Raptis A, Torrejon-Escribano B, Gomez de Aranda I, Blasi J: Distribution of synaptobrevin/VAMP 1 and 2 in rat brain. J Chem Neuroanat 2005, 30:201-211.

14. Tafoya LC, Mameli M, Miyashita T, Guzowski JF, Valenzuela CF, Wilson MC: Expression and function of SNAP-25 as a universal SNARE component in GABAergic neurons. J Neurosci 2006, 26:7826-7838.

15. Garbelli R, Inverardi F, Medici V, Amadeo A, Verderio C, Matteoli M, Frassoni C: Heterogeneous expression of SNAP-25 in rat and human brain. J Comp Neurol 2008, 506:373-386.

16. Zeng Q, Tran T, Tan HX, Hong W: The cytoplasmic domain of VAMP-4 and VAMP-5 is responsible for their correct subcellular targeting. the Nterminal extension of VAMP- 4 contains a dominant autonomous targeting signal for the trans-Golgi network. J Biol Chem 2003, 278:23046-23054.

17. Boschert U, O'Shaughnessy C, Dickinson R, Tessari M, Bendotti C, Catsicas S, Pich EM: Developmental and plasticity-related differential expression of two SNAP-25 isoforms in the rat brain. J Comp Neurol 1996, 367:177-193.

18. Steegmaier M, Yang B, Yoo JS, Huang B, Shen M, Yu S, Luo Y, Scheller RH: Three novel proteins of the syntaxin/SNAP-25 family. J Biol Chem 1998, 273:34171-34179.

19. Su Q, Mochida S, Tian JH, Mehta R, Sheng ZH: SNAP-29. a general SNARE protein that inhibits SNARE disassembly and is implicated in synaptic transmission. Proc Natl Acad Sci USA 2001, 98:14038-14043.

20. Sorensen JB, Nagy G, Varoqueaux F, Nehring RB, Brose N, Wilson MC, Neher E: Differential control of the releasable vesicle pools by SNAP- 25 splice variants and SNAP-23. Cell 2003, 114:75-86.

21. Holt M, Varoqueaux F, Wiederhold K, Takamori S, Urlaub H, Fasshauer D, Jahn R: Identification of SNAP-47, a novel Qbc-SNARE with ubiquitous expression. J Biol Chem 2006, 281:17076-17083.

22. Ibaraki K, Horikawa HP, Morita T, Mori H, Sakimura K, Mishina M, Saisu H, Abe T: Identification of four different forms of syntaxin 3. Biochem Biophys Res Commun 1995, 211:997-1005.
23. Bock JB, Lin RC, Scheller RH: A new syntaxin family member implicated in targeting of intracellular transport vesicles. J Biol Chem 1996, 271:17961-17965.

24. Teng FY, Wang Y, Tang BL: The syntaxins. Genome Biology 2001, 2:3012.1-7.

25. Geng L, Boehmerle W, Maeda Y, Okuhara DY, Tian X, Yu Z, Choe CU, Anyatonwu Gl, Ehrlich BE, Somlo S: Syntaxin 5 regulates the endoplasmic reticulum channel-release properties of polycystin-2. Proc Natl Acad Sci USA 2008, 105:15920-15925.

26. Linial M: SNARE proteins - why so many, why so few? J Neurochem 1997, 69:1781-1792.

27. Gerst JE: SNAREs and SNARE regulators in membrane fusion and exocytosis. Cell Mol Life Sci 1999, 55:707-734.

28. Gibbins IL, Jobling P, Teo EH, Matthew SE, Morris JL: Heterogeneous expression of SNAP-25 and synaptic vesicle proteins by central and peripheral inputs to sympathetic neurons. J Comp Neurol 2003, 459:25-43.

29. Delgado-Martinez I, Nehring RB, Sorensen JB: Differential abilities of SNAP25 homologs to support neuronal function. J Neurosci 2007, 27:9380-9391.

30. Langley K, Grant NJ: Are exocytosis mechanisms neurotransmitter specific? Neurochem Int 1997, 31:739-757.

31. Zimmermann $\mathrm{H}$ : Are mechanisms of exocytosis neurotransmitter specific? Neurochem Int 1997, 31:759-761.

32. Sesack SR, Snyder CL: Cellular and subcellular localization of syntaxin-like immunoreactivity in the rat striatum and cortex. Neuroscience 1995, 67:993-1077

33. Frassoni C, Inverardi F, Coco S, Ortino B, Grumelli C, Pozzi D, Verderio C, Matteoli M: Analysis of SNAP-25 immunoreactivity in hippocampal inhibitory neurons during development in culture and in situ. Neuroscience 2005, 131:813-823.

34. Mandolesi G, Vanni V, Cesa R, Grasselli G, Puglisi F, Cesare P, Strata P: Distribution of the SNAP25 and SNAP23 synaptosomal-associated protein isoforms in rat cerebellar cortex. Neuroscience 2009, 164:1084-1096.

35. Storm-Mathisen J, Leknes AK, Bore AT, Vaaland JL, Edminson P, Haug FM, Ottersen OP: First visualization of glutamate and GABA in neurones by immunocytochemistry. Nature 1983, 301:517-520.

36. Somogyi P, Halasy K, Somogyi J, Storm-Mathisen J, Ottersen OP: Quantification of immunogold labelling reveals enrichment of glutamate in mossy and parallel fibre terminals in cat cerebellum. Neuroscience 1986, 19:1045-1050.

37. Clements JR, Monaghan PL, Beitz AJ: An ultrastructural description of glutamate-like immunoreactivity in the rat cerebellar cortex. Brain Res 1987, 421:343-348.

38. Ottersen OP, Laake JH, Storm-Mathisen J: Demonstration of a releasable pool of glutamate in cerebellar mossy and parallel fibre terminals by means of light and electron microscopic immunocytochemistry. Arch Ital Biol 1990, 128:111-125.

39. Bellocchio EE, Reimer RJ, Fremeau RT Jr, Edwards RH: Uptake of glutamate into synaptic vesicles by an inorganic phosphate transporter. Science 2000, 289:957-960

40. Fremeau RT, Voglmaier S, Seal RP, Edwards RH: VGLUTs define subsets of excitatory neurons and suggest novel roles for glutamate. Trends Neurosci 2004, 27:98-103.

41. Mugnaini E, Oertel WH: An atlas of the distribution of GABAergic neurons and terminals in the rat CNS as revealed by GAD immunohistochemistry. In Handbook of chemical neuroanatomy. Volume 4. GABA and neuropeptides in the CNS, part I. Edited by Bjorklund A, Hokfelt T. British Vancouver: Elsevier; 1985:436-608.

42. Benagiano V, Virgintino D, Rizzi A, Flace P, Troccoli V, Bormann J, Monaghan P, Robertson D, Roncali L, Ambrosi G: Glutamic acid decarboxylase-positive neuronal cell bodies and terminals in the human cerebellar cortex. Histochem J 2000, 32:557-564.

43. Mugnaini E: GABAergic inhibition in the cerebellar system. In GABA in the nervous system: the view at fifty years. Edited by: Martin DL, Olsen RW. Philadelphia: Lippincott Williams 2000:383-407.

44. Benagiano V, Roncali L, Virgintino D, Flace P, Errede M, Rizzi A, Girolamo F, Robertson D, Bormann J, Ambrosi G: GABA immunoreactivity in the human cerebellar cortex. a light and electron microscopical study. Histochem J 2001, 33:537-543

45. Nunzi MG, Birnstiel S, Bhattacharyya BJ, Slater NT, Mugnaini E: Unipolar brush cells form a glutamatergic projection system within the mouse cerebellar cortex. J Comp Neurol 2001, 434:329-341. 
46. Flace $P$, Benagiano V, Lorusso L, Girolamo F, Rizzi A, Virgintino D, Roncali L, Ambrosi G: Glutamic acid decarboxylase immunoreactive large neuron types in the granular layer of the human cerebellar cortex. Anat Embryol 2004, 208:55-64.

47. Ambrosi G, Flace P, Lorusso L, Girolamo F, Rizzi A, Bosco L, Errede M, Virgintino $D$, Roncali $L$, Benagiano $V$ : Non-traditional large neurons in the granular layer of the cerebellar cortex. Eur J Histochem 2007, 51:59-64.

48. Honer WG, Hu L, Davies P: Human synaptic proteins with a heterogeneous distribution in cerebellum and visual cortex. Brain Res 1993, 609:9-20.

49. Hong RM, Mori H, Fukui T, Moriyama Y, Futai M, Yamamoto A, Tashiro Y, Tagaya M: Association of $\mathrm{N}$-ethylmaleimide-sensitive factor with synaptic vesicles. FEBS Lett 1994, 350:253-257.

50. Yan XX, Jen LS, Garey LJ: Spatial periodicity of NADPH-diaphorase and synaptophysin, but not SNAP-25, reactivity in the monkey cerebellar cortex. Brain Res 1995, 669:285-290.

51. Oyler GA, Polli JW, Wilson MC, Billingsley ML: Development expression of the 25-kDa synaptosomal-associated protein (SNAP-25) in rat brain. Proc Natl Acad Sci USA 1999, 88:5247-5251.

52. Nishiki T, Nihonmatsu I, Tsuhara Y, Kawasaki M, Sekiguchi M, Sato K, Mizoguchi A, Takahashi M: Distribution of soluble N-ethylmaleimide fusion protein attachment proteins (SNAPs) in the rat nervous system. Neuroscience 2001, 107:363-371.

53. Tafoya LC, Shuttleworth WC, Yanagawa Y, Obata K, Wilson MC: The role of the t-SNARE SNAP 25 in action potential-dependent calcium signaling and expression in GABAergic and glutamatergic neurons. BMC Neuroscience 2008, 9:105:1-17.

54. Matteoli M, Pozzi D, Grumelli C, Condliffe SB, Frassoni C, Harkany T, Verderio C: The synaptic split of SNAP-25. different roles in glutamatergic and GABAergic neurons? Neuroscience 2009, 158:223-230.

55. Schoch S, Deak F, Konigstorfer A, Mozhayeva M, Sara Y, Sudhof TC, Kavalali ET: SNARE function analyzed in synaptobrevin/VAMP knockout mice. Science 2001, 294:1117-1122.

56. Deak F, Schoch S, Liu X, Sudhof TC, Kavalali ET: Synaptobrevin is essential for fast synaptic-vesicle endocytosis. Nat Cell Biol 2004, 11:1102-1108.

57. Miya F, Yamamoto A, Akagawa K, Kawamoto K, Tashiro Y: Localization of HPC-1/syntaxin 1 in developing rat cerebellar cortex. Cell Struct Funct 1996, 21:525-532.

58. Kaneko T, Fujiyama F, Hioki H: Immunohistochemical localization of candidates for vesicular glutamate transporters in the rat brain. $J$ Comp Neurol 2002, 444:39-62.

59. Hioki H, Fujiyama F, Taki K, Tomioka R, Furuta T, Tamamaki N, Kaneko T: Differential distribution of vesicular glutamate transporters in the rat cerebellar cortex. Neuroscience 2003, 117:1-6.

60. Hisano S, Sawada K, Kawano M, Kanemoto M, Xiong G, Mogi K, SakataHaga H, Takeda J, Fukui Y, Nogami H: Expression of inorganic phosphate/ vesicular glutamate transporters (BNPI/VGLUT1 and DNPI/VGLUT2) in the cerebellum and precerebellar nuclei of the rat. Brain Res Mol Brain Res 2003, 107:23-31.

61. Benagiano V, Lorusso L, Flace P, Girolamo F, Rizzi A, Bosco L, Ambrosi G: Heterogeneity of mossy fibre terminals in the granular layer of rat cerebellar cortex as revealed by immunohistochemistry for vGluT-1, vGluT-2 and SNAP-25. Eur J Histochem 2011, 55(supplement 1):30.

62. Gebre SA, Reeber SL, Sillitoe RV: Parasagittal compartmentation of cerebellar mossy fibers as revealed by patterned expression of vesicular glutamate transporters VGLUT1 and VGLUT2. Brain Struct Funct 2011.

63. Benagiano V, Lorusso L, Coluccia A, Tarullo A, Flace P, Girolamo F, Bosco L, Cagiano R, Ambrosi G: GAD and GABA immunoreactivities in the cerebellar cortex of adult rat after prenatal exposure to a low concentration of carbon monoxide. Neuroscience 2005, 135:897-905.

64. Cubelos B, Gimenez C, Zafra F: Localization of the GLYT1 glycine transporter at glutamatergic synapses in the rat brain. Cereb Cortex 2005, 15:448-459.

65. Wouterlood FG, Canto CB, Aliane V, Boekel AJ, Grosche J, Härtig W, Beliën JA, Witter MP: Coexpression of vesicular glutamate transporters 1 and 2, glutamic acid decarboxylase and calretinin in rat entorhinal cortex. Brain Struct Funct 2007, 212:303-319.

66. Garcia EP, Mc Pherson PS, Chilcote TJ, Takei K, De Camilli P: rbSec1A and B colocalize with syntaxin 1 and SNAP-25 throughout the axon, but are not in a stable complex with syntaxin. J Cell Biol 1995, 129:105-120.
67. Galli T, Garcia EP, Mundigl O, Chilcote TJ, De Camilli P: v-and t-SNAREs in neuronal exocytosis. a need for additional components to define sites of release. Neuropharmacology 1995, 34:1351-1360.

68. Wang $Y$, Tang BL: SNAREs in neurons-beyond synaptic vesicle exocytosis. Mol Membr Biol 2006, 23:377-384.

69. Bragina L, Candiracci C, Barbaresi P, Giovedi S, Benfanti F, Conti F: Heterogeneity of glutamatergic and GABAergic release machinery in cerebral cortex. Neuroscience 2007, 146:1829-1840.

70. Bragina L, Giovedì S, Barbaresi P, Benfanti F, Conti F: Heterogeneity of glutamatergic and GABAergic release machinery in cerebral cortex. analysis of synaptogyrin, vesicle- associated membrane protein, and Syntaxin. Neuroscience 2010, 165:934-943.

71. Bergersen LH, Gundersen V: Morphological evidence for vesicular glutamate release from astrocytes. Neuroscience 2009, 158:260-265.

\section{doi:10.1186/1471-2202-12-118}

Cite this article as: Benagiano et al:: VAMP-2, SNAP-25A/B and syntaxin1 in glutamatergic and GABAergic synapses of the rat cerebellar cortex. BMC Neuroscience 2011 12:118.

\section{Submit your next manuscript to BioMed Central and take full advantage of:}

- Convenient online submission

- Thorough peer review

- No space constraints or color figure charges

- Immediate publication on acceptance

- Inclusion in PubMed, CAS, Scopus and Google Scholar

- Research which is freely available for redistribution

Submit your manuscript at www biomedcentral com/submit
Ciomed Central 